\title{
Divisi: Learning from Semantic Networks and Sparse SVD
}

\author{
Rob Speer ${ }^{\ddagger *}$, Kenneth Arnold ${ }^{\S}$, Catherine Havasi ${ }^{\S}$
}

\begin{abstract}
Singular value decomposition (SVD) is a powerful technique for finding similarities and patterns in large data sets. SVD has applications in text analysis, bioinformatics, and recommender systems, and in particular was used in many of the top entries to the Netflix Challenge. It can also help generalize and learn from knowledge represented in a sparse semantic network.

Although this operation is fundamental to many fields, it requires a significant investment of effort to compute an SVD from sparse data using Python tools. Divisi is an answer to this: it combines NumPy, PySparse, and an extension module wrapping SVDLIBC, to make Lanczos' algorithm for sparse SVD easily usable within cross-platform Python code.

Divisi includes utilities for working with data in a variety of sparse formats, including semantic networks represented as edge lists or NetworkX graphs. It augments its matrices with labels, allowing you to keep track of the meaning of your data as it passes through the SVD, and it can export the labeled data in a format suitable for separate visualization GUls.
\end{abstract}

Index Terms-SVD, sparse, linear algebra, semantic networks, graph theory

\section{Introduction}

Singular value decomposition (SVD) is a way of factoring an arbitrary rectangular matrix, in order to express the data in terms of its principal components. SVD can be used to reduce the dimensionality of a large matrix, a key step in many domains, including recommender systems, text mining, search, statistics, and signal processing.

The truncated $S V D$, in which only the largest principal components are calculated, is a particularly useful operation in many fields because it can represent large amounts of data using relatively small matrices. In many applications, the input to the truncated SVD takes the form of a very large, sparse matrix, most of whose entries are zero or unknown.

Divisi provides the Lanczos algorithm [Lan98] for performing a sparse, truncated SVD, as well as useful tools for constructing the input matrix and working with the results, in a reusable Python package called divisi2. It also includes important operations for preparing data such as normalization and mean-centering. More experimentally, Divisi also includes implementations of some SVD-inspired algorithms such as CCIPCA [Wen03] and landmark multi-dimensional scaling [Si104].

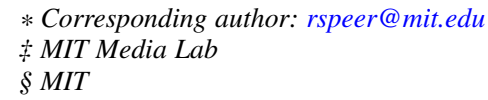

Copyright $(2010$ Rob Speer et al. This is an open-access article distributed under the terms of the Creative Commons Attribution License, which permits unrestricted use, distribution, and reproduction in any medium, provided the original author and source are credited.
Using singular value decomposition, any matrix $A$ can be factored into an orthonormal matrix $U$, a diagonal matrix $\Sigma$, and an orthonormal matrix $V^{T}$, so that $A=U \Sigma V^{T}$. The singular values in $\Sigma$ can be ordered from largest to smallest, where the larger values correspond to the vectors in $U$ and $V$ that are more significant components of the initial $A$ matrix. The largest singular values, and their corresponding rows of $U$ and columns of $V$, represent the principal components of the data.

To create the truncated SVD, discard all but the first $k$ components-the principal components of $A$-resulting in the smaller matrices $U_{k}, \Sigma_{k}$, and $V_{k}^{T}$. The components that are discarded represent relatively small variations in the data, and the principal components form a low-rank approximation of the original data. One can then reconstruct a smoothed version of the input matrix as an approximation: $A \approx U_{k} \Sigma_{k} V_{k}^{T}=A_{k}$.

To make it easier to work with SVD in understandable Python code, Divisi provides an abstraction over sparse and dense matrices that allows their rows and columns to be augmented with meaningful labels, which persist through various matrix operations.

The documentation for installing and using Divisi is hosted at http://csc.media.mit.edu/docs/divisi2/.

\section{Architecture}

Divisi is built on a number of other software packages. It uses NumPy [Oli10] to represent dense matrices, and PySparse [Geu08] to represent sparse ones, and uses a Cython wrapper around SVDLIBC [Roh10] to perform the sparse SVD. It can optionally use NetworkX [Net10] to take input from a directed graph such as a semantic network.

Divisi works with data in the form of labeled arrays. These arrays can be sparse or dense, and they can be 1-D vectors or 2-D matrices.

Figure 1 shows the relationships between classes in Divisi2. The yellow-highlighted classes are the ones that are intended to be instantiated. The core representations use multiple inheritance: for example, the properties of a SparseMatrix are separately defined by the fact that it is sparse and the fact that it is a 2-D matrix.

Sparse arrays encapsulate a PysparseMatrix from the pysparse package, while dense arrays are a subclass of numpy.ndarray and therefore support most NumPy operations. Both representations support NumPy-style "fancy indexing".

A vector contains a single, optional list of labels: if it exists, each entry in the list corresponds to one entry in the vector. A matrix may have two lists of labels: one assigns a label to each 


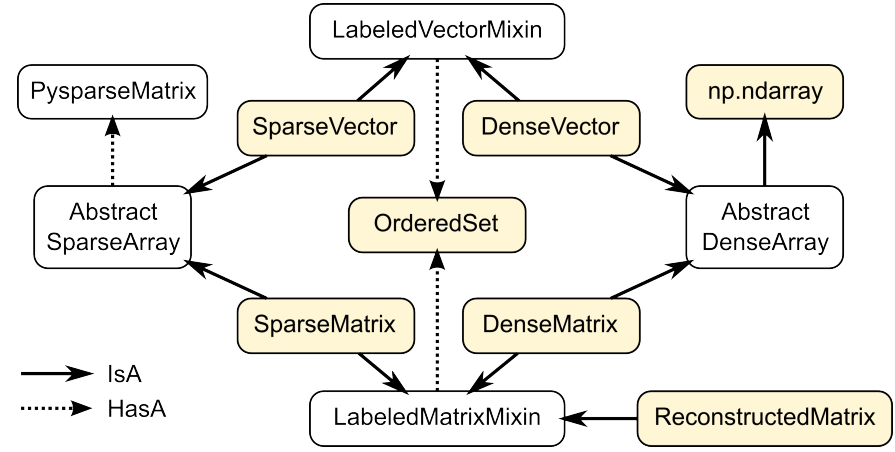

Fig. 1: Relationships between the main classes in Divisi 2.0, as well as some externally-defined classes.

row, and another assigns a label to each column. The purpose of these labels is to associate a meaning with each entry in a vector or matrix, so that code can look up entries by what they mean instead of simply by their position in the matrix.

The label lists themselves are instances of Divisi's OrderedSet class, which augments a list with a dictionary of its values, so that it can perform the .index () operation-finding an entry by its value-in constant time. This enables methods such as SparseMatrix.row_named(label), efficiently returning the row with a particular label.

One more important class is ReconstructedMatrix, which lazily evaluates the matrix product of two DenseMatrices. This allows using the SVD as an approximation to a large matrix, but stores that large approximate matrix as a product of its SVD factors instead, which requires much less memory.

Next, we will explain the features of Divisi through three examples: performing latent semantic analysis (LSA) over documents from the Brown corpus, making movie recommendations from a MovieLens data set, and drawing conclusions based on ConceptNet (a semantic network of general knowledge).

\section{Latent semantic analysis}

One common use for Divisi is to make a topic model from a number of documents using latent semantic analysis (LSA). LSA typically consists of constructing a bag-of-words matrix of the words that appear in various documents, normalizing that matrix using tf-idf, and taking the SVD.

We'll use as an example a collection of 44 documents from the "news" section of the Brown corpus, a sample of which is available through NLTK:

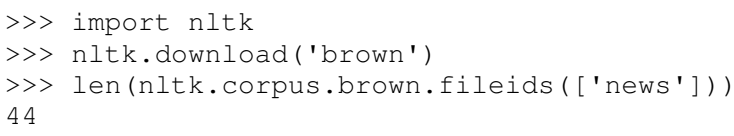

When searching for articles about, say, books, we don't generally care whether the document contained "book" or "books". NLTK includes the Porter stemmer, which strips off endings:

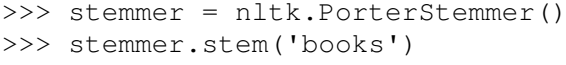

In the most basic form of LSA, each document is treated as a "bag of words", ignoring all sequence and punctuation. The following function yields all the stemmed words from a document in the Brown corpus:

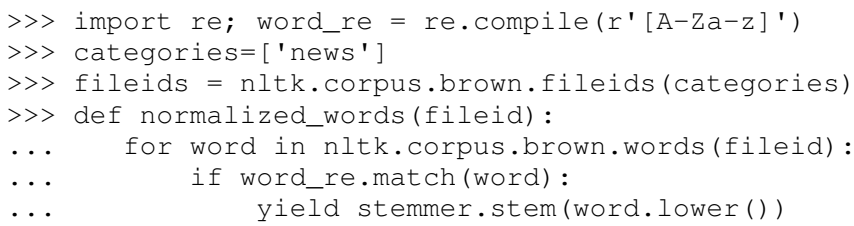

Now that we have the input data, we can load it into a Divisi sparse matrix. The function divisi2. make_sparse ${ }^{1}$ creates a sparse matrix from a list of entries, each of which is a tuple of (value, row, col):

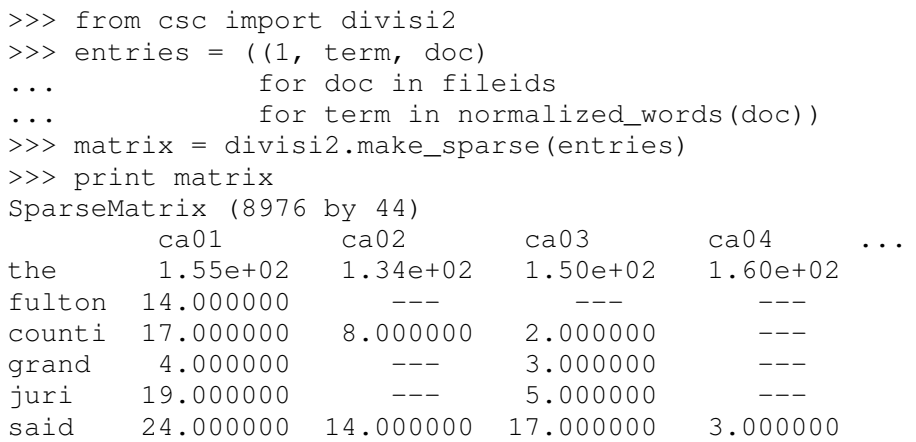

...

A Divisi sparse matrix behaves like a NumPy array, but has additional facilities for labeling entries. Notice that row and $\mathrm{COl}$ were both specified as strings (a term and a filename) rather than numbers. The row_labels and $\mathrm{COl}_{\text {_l }}$ labels attributes keep track of what label is assigned to each row or column index: ${ }^{2}$ :

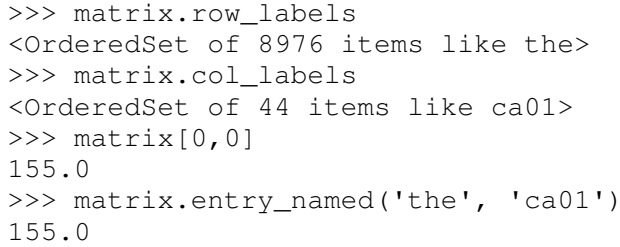

That entry indicates that the word "the" appeared 155 times in the first document alone. Such common words would overwhelm the analysis: we should give less weight to words that appear in nearly every document. Also, a document that is twice as long as average should not necessarily be twice as influential. The standard solution to these problems is called $t f-i d f$ normalization and is one of several normalization capabilities provided by Divisi:

$>>$ normalized = matrix.normalize_tfidf().squish ()

All Divisi normalization routines return a copy of their input. The final .squish() call deals with words like "the": since they appear in every document, their idf value, and thus the value of every entry in the corresponding row, is 0 . Rows and columns that are all zeros leave part of the SVD result unconstrained, so we remove them for numerical stability.

Next we can compute the SVD. The only parameter is the number of singular values ("components") to keep. The optimal value depends on the corpus and task at hand; it essentially controls how much you want to fill in gaps in your data. Since the corpus is small, we arbitrarily choose 10 for this example.

$\gg>$ u, sigma, $v=$ normalized.svd $(k=10)$

1. The version of Divisi described in this paper, Divisi 2.0, would be installed in a namespace package called Csc. Divisi 2.2 can now be imported directly as divisi2, but references to csc.divisi2 still work.

2. Example output in this paper is truncated or rounded for brevity. 
Here, sigma is an array of diagonal entries; the actual diagonal matrix $\Sigma$ is given by np.diag (sigma).

Since $A \approx U \Sigma V^{T}$, we can execute various queries simply by matrix multiplication. For example, which documents are likely to contain terms like "book"? That's just a row of $A$. Using the approximation, we can compute that row:

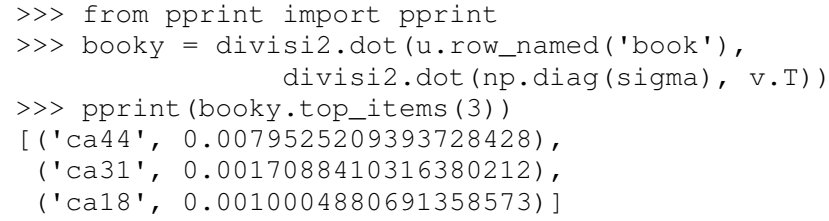

divisi2.dot is a wrapper around numpy.dot that ensures that labels are maintained properly.

\section{Reconstructing an approximate matrix}

Divisi provides simpler ways of working with matrix reconstructions: the ReconstructedMatrix class:

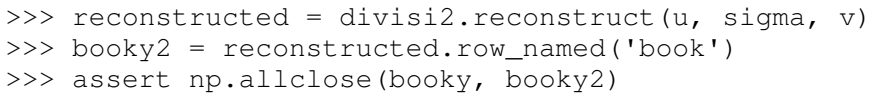

Another common query, often seen in blog posts, is which articles are similar to the one in question. Mathematically, which other document has the term vector with the highest dot product with the term vector of this document? The answer is again found in a matrix slice, this time of

$$
A^{T} A=V \Sigma U^{T} U \Sigma V^{T}=V \Sigma^{2} V^{T} .
$$

Again, Divisi provides functionality for easily slicing similarity matrices:

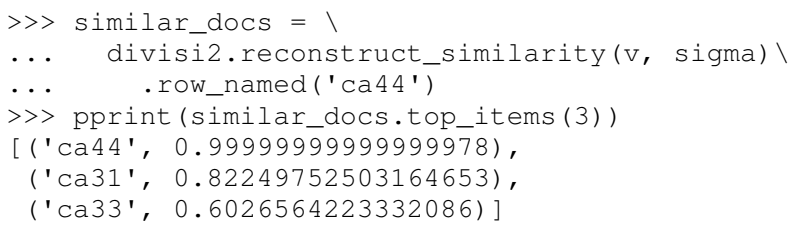

By default, reconstruct_similarity normalizes the result values to lie between -1 and 1 .

\section{Making recommendations}

In the above example, we assumed that unspecified entries in the input matrix were zero, representing a lack of knowledge. When using SVD over a data set whose numeric values do not meaningfully start at zero, some adjustments are necessary.

In the domain of movie recommendations, for example, the input data often takes the form of star ratings that people assign to movies, ranging from 1 to 5. A 5-star rating and a 1-star rating are as different as can be, so a 5-star rating certainly does not have the meaning of "a 1-star rating, but five times more so".

In fact, the scale of ratings differs among people and movies. A movie rater may be very stingy with high ratings, so if they give a movie five stars it is very meaningful. Likewise, a movie could be widely panned, receiving a 1.1 star rating on average, so when someone gives the movie five stars it says that there is something very different about their taste in movies.

The movie rating problem can be broken down into two steps [Kor09]: accounting for the biases in ratings inherent to each movie and each person, and learning how people's particular preferences differ from those biases. We can represent the second step as an SVD where zero does represent a lack of information, and add the biases back in when we reconstruct the matrix.

To begin the example, load the MovieLens dataset of 100,000 movie ratings [Kon98], which is provided free from http:// grouplens.org:

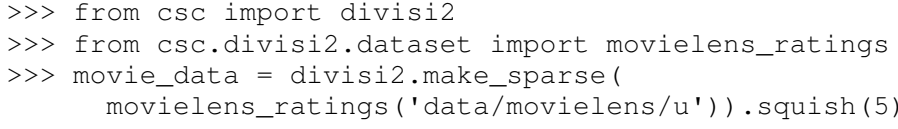

The "squish" method at the end discards users and movies with fewer than 5 ratings.

With this data, for example, we can query for the movies with the highest row bias (and therefore the highest average rating):

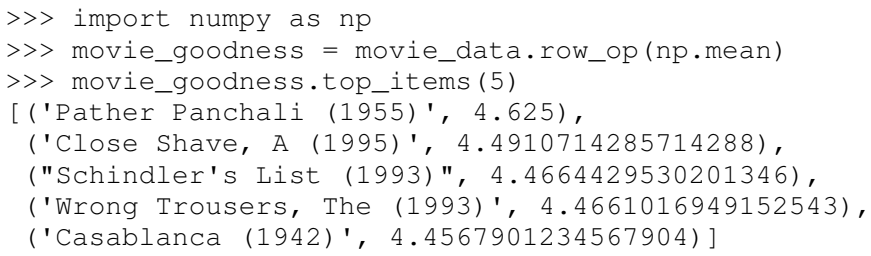

We use the SparseMatrix.mean_center() method to remove the biases, leaving only the differences from the mean, calculate a 20-dimensional truncated SVD from those differences, and reconstruct an approximate matrix that predicts people's movie ratings.

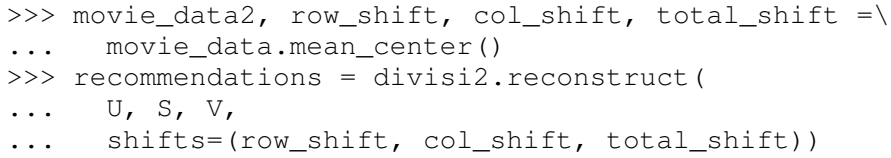

Let's look in particular at user number 5, who rated 174 movies. We can get a vector of their recommendations and query for the best ones:

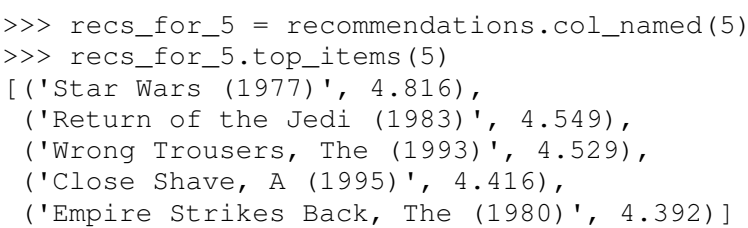

We see that this user should really like the Star Wars Trilogy, but this is unsurprising because the user in fact already told MovieLens they liked those movies. To get true recommendations, we should make sure to filter for movies they have not yet rated.

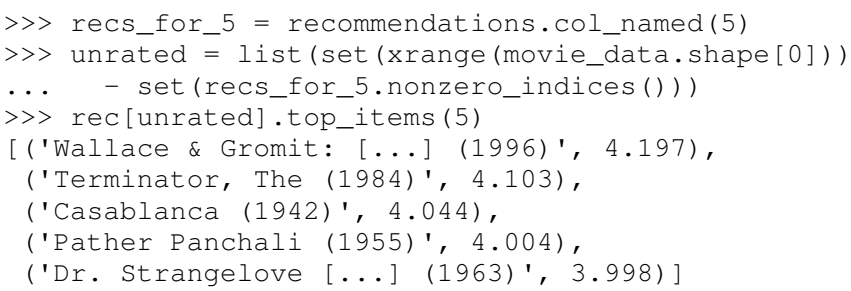

And on the other end of the scale, if we look for the best antirecommendation in (-rec [unrated]), we find that user 5 should give "3 Ninjas: High Noon At Mega Mountain" a rating of 0.24 stars.

SVD alone does not make a cutting-edge, high-quality recommender system, but it does a reasonable part of the job. This process has been used as a component of many recommender systems, including the Netflix Prize-winning system, Bellkor's Pragmatic Chaos [Kor09], and Divisi makes it easy to do in Python. 


\section{Learning from a semantic network}

Divisi contains methods for learning from data in a semantic network in NetworkX format. The network can contain labeled nodes and labeled edges with weights on each edge, and can build matrices that relate these to each other in a variety of ways.

This is an important feature of Divisi, because it extends its scope to data that is not traditionally represented as a matrix. It can learn from and generalize patterns that appear in any semantic network, and it is especially effective if that network contains redundancies or incomplete information. For this reason, we often use it to learn from ConceptNet [Hav07], a network of people's general "common sense" knowledge about the real world. A graph representation of ConceptNet 4.0 is included with Divisi 2.0.

The divisi2. network module defines the various ways to extract information from these labeled semantic networks. Its sparse_triples() function turns the list of edges into a list of (value, rowlabel, columnlabel) triples that can be used to build a sparse matrix, and uses the arguments row_labeler and col_labeler to specify how the values are assigned to labels. sparse_matrix () goes the extra step to turn these triples into a matrix.

In many cases, the labeler will give two results for each edge, because each edge connects two nodes. When the row and column labelers both give two results, they will be paired up in contrary order. The next example will clarify why this is useful.

One simple labeler is 'nodes', which extracts the source and target nodes of each edge. If an edge of weight 1 connects "dog" to "bark", then because of the contrary order rule, sparse_matrix(graph, 'nodes', 'nodes') will put a 1 in the entry whose row is "dog" and column is "bark", as well as the entry whose row is "bark" and whose column is "dog". The resulting overall matrix is the adjacency matrix of the graph.

'features' is a more complex labeler: it takes the edge label into account as well, and describes an incoming or outgoing edge, including the node on the other side of it. The idea is that a node can be combined with a feature to completely describe an edge.

For example, consider a weight-1 edge from "dog" to "mammal", labeled with "IsA", expressing the assertion that "a dog is a mammal". The matrix sparse_matrix (graph, 'nodes', ' features' ) will then express both the fact that the node "dog" has the feature "IsA mammal", and that "mammal" has the feature "dog IsA".

These features are represented with Divisi as 3-tuples of (direction, edge label, node label), where direction is "left" or "right" depending on whether this is an incoming or outgoing edge.

Other possible labelers are "relations", which extracts just the edge label, and "pairs", extracting the source and target nodes as tuples, and more can be defined as functions.

The process called AnalogySpace [Spe08] involves making a node vs. feature matrix of common sense knowledge and generalizing it with a truncated SVD. We will show an example of doing this with ConceptNet here.

\section{Learning from ConceptNet}

Start by loading the pre-defined ConceptNet 4.0 graph:

\footnotetext{
>> conceptnet_graph = divisi2.load (

'data:graphs/conceptnet_en.graph' )
}

We can break this graph down into nodes and features, and see a sample of what it looks like:

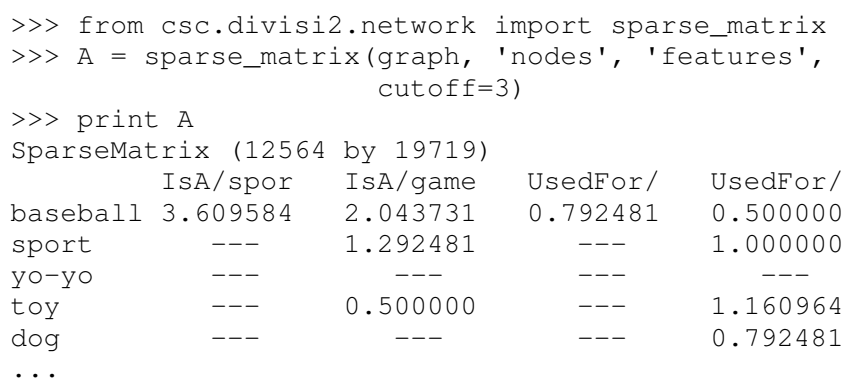

And with that, we can make a truncated SVD and reconstruct an approximation to $\mathrm{A}$ :

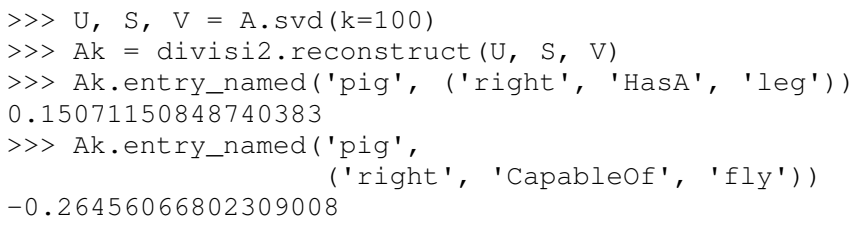

As shown in the earlier LSA example, we can also reconstruct an approximation to the similarity matrix $A^{T} A$, describing how similar the nodes are to each other:

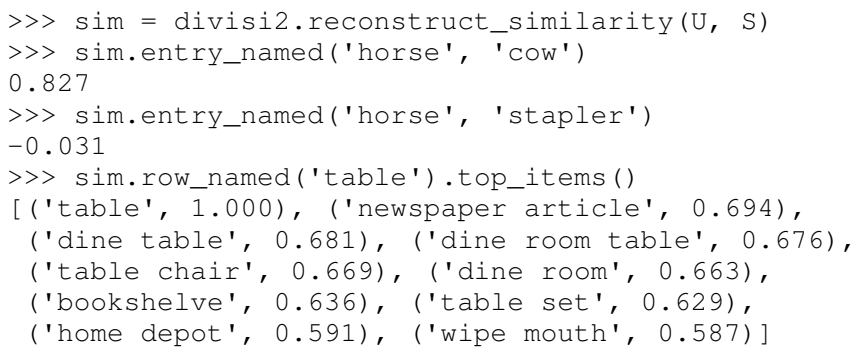

Recall that reconstruct_similarity normalizes its values to between -1 and 1 . Here, this normalization makes some nodes, such as "newspaper article" and "home depot", get a spuriously high weight because their truncated SVD vectors had low magnitude. When ranking possible similarities-or, for that matter, predictions for new assertions that could be true-we have found it more useful to normalize the vectors to unit vectors before the SVD, so that nodes that are weakly described by the SVD do not end up magnified.

Divisi allows for pre-SVD normalization with the SparseMatrix methods normalize_rows (), normalize_cols (), and normalize_all(). (tf-idf normalization, like in the LSA example, is also an option, but it is inappropriate here because it de-emphasizes common concepts.) The first two scale the rows or columns, respectively, of the input so that they become unit vectors. However, normalizing the rows can further distort the magnitudes of the columns, and vice versa, and there is no way to exactly normalize both the rows and columns of an arbitrary matrix.

We have found that a compromise works best: normalize each entry by the geometric mean of its row and column magnitudes. This is what SparseMatrix.normalize_all() does, and we favor it in this case because not only does it put all the rows and columns on approximately the same scale, it also increases the predictive accuracy of the reconstructed SVD (which we will be able to quantify in a moment).

In this representation, we can look again at the similarities for "table": 


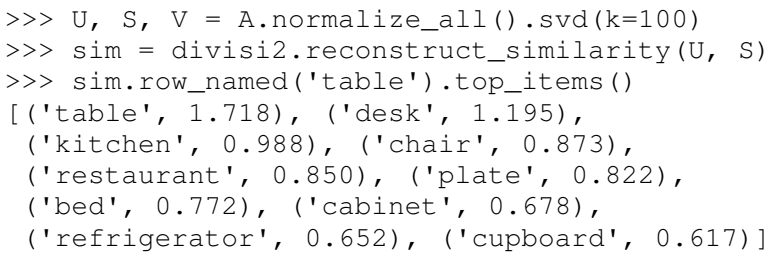

\section{Choosing parameters}

So far, we have used two parameters in this process without justification: the method of normalization, and the value of $k$.

Instead of simply tweaking these parameters by hand, we can bring in some test data and search for the parameters that maximize the predictive value of the SVD. Because what we care about is the relative ranking of statements, not the numerical values they are assigned, a traditional mean-squared evaluation does not exactly make sense.

However, using Divisi, we can evaluate how often the relative ranking of a pair of assertions agrees with the ranking that a human would give them. In the case of ConceptNet, we have already acquired many such human-evaluated statements from evaluations such as the one in [Spe08], so we use those as the source of goldstandard rankings.

The ReconstructedMatrix.evaluate_ranking() method is what we use to compare pairwise rankings in this way. We can use it, first of all, to confirm that normalize_all () performs better than the other possible normalization methods on ConceptNet, leaving $k$ fixed at 100 . The results are:

- Without normalization: $68.47 \%$ agreement

- Using normalize_rows: $67.66 \%$ agreement

- Using normalize_cols: $67.30 \%$ agreement

- Using normalize_all: 70.77\% agreement

Then, after applying that normalization method, we can try truncated SVDs with various values of $k$.

$>>>$ from csc.divisi2.network import conceptnet_matrix

$\gg$ conceptnet $=$ conceptnet_matrix('en').normalize_all()

$>>$ testdata = divisi2.load('usertest_data.pickle')

$>>$ accuracy_data $=[$ ]

$\gg$ for $k$ in xrange $(1,200)$ :

$\ldots \quad \mathrm{U}, \mathrm{S}, \mathrm{V}=$ conceptnet. $\mathrm{svd}(\mathrm{k}=\mathrm{k})$

... rec $=$ divisi2.reconstruct $(U, S, V)$

... correct, total, accuracy $=\backslash$

... rec.evaluate_ranking (testdata)

... accuracy_data.append (accuracy)

The plot of the resulting accuracy_data in Figure 2 shows a plateau of good values of $k$, roughly between $k=100$ and $k=200$.

\section{Memory use and scalability}

The main use case of Divisi2 is to decompose a sparse matrix whose entries fit in memory. The objects that primarily consume memory are:

- The linked lists that comprise the PySparse matrix

- The compressed-sparse-column copy of this matrix used by SVDLIBC

- The dense matrices $\mathrm{U}$ and $\mathrm{V}$, and the vector $\mathrm{S}$, that are returned by SVDLIBC and used directly by NumPy

- The optional OrderedSets of labels (each using a Python list and dictionary)

Each nonzero entry in a sparse matrix and each entry in a dense matrix requires the space of a $\mathrm{C}$ double (assumed to be 8 bytes).

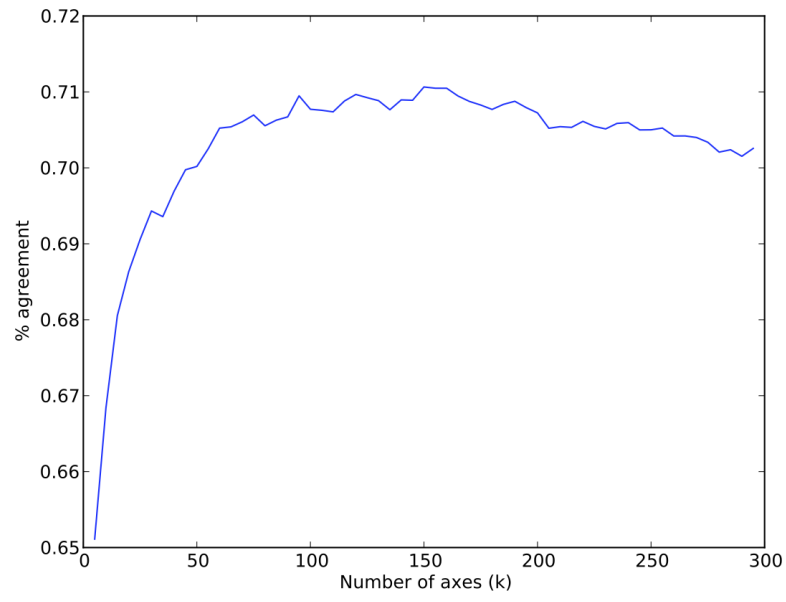

Fig. 2: Evaluating the predictive accuracy of the truncated SVD on ConceptNet for various values of $\mathrm{k}$.

The PySparse matrix also requires an integer (4 bytes), acting as a pointer, for each entry. (This implementation incidentally limits matrices to having fewer than $2^{31}$ nonzero entries.) The non-zero entries in the compressed-sparse-column matrix also come with integer row numbers. Finally, each allocated row requires two integer pointers.

So, without labels, a rank $k$ decomposition of an $m \times n$ matrix with $z$ non-zero entries requires $(24 z+8 m+8 k(m+n))$ bytes, plus a negligible amount of overhead from Python and $C$ structures. As a practical example, it is possible within the $4 \mathrm{GiB}$ memory limit of 32-bit CPython to take a rank-100 decomposition of a $10^{6} \times 10^{6}$ matrix with $10^{8}$ entries, or a rank-10 decomposition of a $10^{7} \times 10^{7}$ matrix with $10^{8}$ entries, each of which requires 3.7 to $3.8 \mathrm{GiB}$ plus overhead.

In order to support even larger, denser data sets, Divisi 2.2 includes an experimental implementation of Hebbian incremental SVD that does not require storing the sparse data in memory.

\section{Conclusion}

The SVD is a versatile analysis tool for many different kinds of data. Divisi provides an easy way to compute the SVD of large sparse datasets in Python, and additionally provides Pythonic wrappers for performing common types of queries on the result.

Divisi also includes a variety of other functionality. For example, it can analyze combinations of multiple matrices of data, a technique called blending, which is useful for drawing conclusions from multiple data sources simultaneously.

Further documentation about Divisi2, including the presentation from SciPy 2010, is available at http://csc.media.mit.edu/ docs/divisi2/.

\section{REFERENCES}

[Kor09] Y. Koren, R. Bell, and C. Volinsky. Matrix Factorization Techniques for Recommender Systems. Computer, 42(8):30-37, August 2009.

[Kon98] J. Konstan, J. Riedl, A. Borchers, and J. Herlocke. Recommender Systems: A GroupLens Perspective. Papers from the 1998 Workshop on Recommender Systems, Chapel Hill, NC. 1998.

[Net10] NetworkX Developers. NetworkX. Viewable online at:http://networkx.lanl.gov/, 2010.

[Roh10] Doug Rohde. SVDLIBC. Viewable online at: http://tedlab.mit.edu/ $\sim \mathrm{dr} / \mathrm{SVDLIBC} /, 2010$

[Geu08] Roman Geus, Daniel Wheeler, and Dominique Orban. PySparse Viewable online at: http://pysparse.sourceforge.net/, 2008. 
[Oli10] Travis Oliphant. Guide to Numpy. Viewable online at: http://www. tramy.us/, 2010.

[Sil04] Vin de Silva and Joshua B. Tenenbaum. Sparse multidimensional scaling using landmark points. Stanford University Technical Report, 2004.

[Wen03] Juyang Weng and Yilu Zhang and Wey-Shiuan Hwang. Candid covariance-free incremental principal component analysis. IEEE Transactions on Pattern Analysis and Machine Intelligence, 25(8):1034-1040, August 2003.

[Lan98] Cornelius Lanczos and William R. Davis (ed). Collected published papers with commentaries. North Carolina State University, 1998.

[Hav07] Catherine Havasi, Robert Speer, and Jason Alonso. ConceptNet 3: a Flexible, Multilingual Semantic Network for Common Sense Knowledge. Recent Advances in Natural Language Processing, September 2007.

[Spe08] Robert Speer and Catherine Havasi and Henry Lieberman. AnalogySpace: Reducing the Dimensionality of Common Sense Knowledge. Proceedings of AAAI 2008, July 2008. 